\title{
Repetition blindness is orientation blind
}

\author{
Michael C. Corballis and Cole Armstrong \\ University of Auckland, Auckland, New Zealand
}

\begin{abstract}
In identifying rapid sequences of three letters, subjects were worse at identifying the first and third letters when they were the same than when they were different, indicating repetition blindness (RB). This effect occurred regardless of the angular orientations of the letters, but was more pronounced when the orientations of the repeated letters were different than when they were the same. In a second experiment, RB was also evident when the first and third letters were lowercase bs or ds, presented upright or inverted, even though they are differently named when inverted ( $q$ and $p$, respectively). Conversely, a third experiment showed that RB occurred when the letters had the same names but were repeated in different case. These results suggest that the early extraction of letter shape is independent of its orientation and left-right sense, and that RB can occur at the levels of both shape and name.
\end{abstract}

There has been some debate as to the role of orientation in the recognition of familiar shapes or objects. Some have proposed that shapes or objects are stored in one or more specific orientations relative to the viewer (e.g., Bülthoff \& Edelman, 1992; Palmer, Rosch, \& Chase, 1981; Tarr \& Pinker, 1989), whereas others have suggested that the basic shape of an object is stored independently of its orientation (e.g., Biederman, 1987; Biederman \& Gerhardstein, 1993; Corballis, 1988; Deutsch, 1962). There is some evidence that accuracy or reaction time in recognition tends to fall off with increasing rotation of the object away from a preferred orientation (e.g., Jolicœur, 1985; Murray, 1995), supporting orientationspecific representation, whereas neuropsychological evidence suggests that objects are stored independently of orientation. In particular, patients with a condition known as orientation agnosia can recognize rotated objects but are unable to determine their orientations (Fujinaga, Muramatsu, Ogano, \& Kato, 2005; Harris, Harris, \& Caine, 2001; Karnath, Ferber, \& Bülthoff, 2000; Turnbull, Della Sala, \& Beschin, 2002). Jolicœur (1990) has suggested a dual-process theory in which initial recognition of an object depends on matching to an orientation-dependent representation, but with repeated exposure to the same object, recognition can be achieved independently of orientation through the matching of features. Another possibility is that initial basic-level recognition is independent of orientation, but that observers may then "correct" for rotation away from the upright, perhaps through mental rotation, to provide a more detailed level of recognition (Corballis, 1988).

The phenomenon of repetition blindness (RB) suggests a further means of testing the role of orientation in recognition. RB occurs under conditions of rapid serial visual presentation (RSVP), when observers typically find it more difficult to detect a repeated item if it is presented within about $400 \mathrm{msec}$ of its first presentation than to detect a different item in the same temporal position. One explanation for this is the so-called "token individuation hypothesis" (Kanwisher, 1987), which is that, under RSVP, presentation is too rapid for observers to assign separate tokens to the same type. That is, the second presentation is not registered as a separate episodic event. Nevertheless, the sensitivity to repetition implies that the identity of the repeated item must be extracted, even though it is unreported, implying recognition without awareness (Kanwisher, Yin, \& Wojciulik, 1999). Not all authors agree that RB is due to a dissociation between type and token, as suggested by Kanwisher (1987). Alternative hypotheses are that it reflects a failure of retrieval (e.g., Armstrong \& Mewhort, 1995; Fagot \& Pashler, 1995), online reconstruction (Whittlesea \& Masson, 2005), or integration (Morris \& Harris, 2002).

Previous studies have also shown that RB occurs in the identification of pictures of objects even when the orientations of the repeated objects differ on the two presentations (Harris \& Dux, 2005; Kanwisher et al., 1999). According to the type-token distinction, this implies that the extraction of type is independent of orientation. Alternatively, one might simply suppose that there is failure to bind orientation to identity, whether through online reconstruction, perceptual integration, or retrieval. Whatever the reason, the dissociation between shape and orientation might also be understood, at least in part, in terms of the distinction between the dorsal and ventral streams in the cortical visual system, as identified by Ungerleider and Mishkin (1982). The ventral system, extending into the inferior temporal cortex, may be responsible for the recognition of shape, independently of

M. C. Corballis, m.corballis@auckland.ac.nz 
orientation, whereas the dorsal system, extending into the parietal lobes, may indicate how objects are located and oriented in space. The dorsal system presumably does not "know" the actual identity of a given object, but it nevertheless registers the regions of 3-D space that it occupies. It has been shown, for example, that patients with object agnosia due to damage to the ventral system may nevertheless understand the shape of an object well enough to be able to insert it into an appropriately shaped slot (Milner \& Goodale, 1995).

In the present study, we examine further the effect of orientation on RB for familiar shapes - in this case, letters. In the study by Kanwisher et al. (1999), the stimuli were rotated only up to $30^{\circ}$, whereas in the study by Harris and Dux (2005), one of the two repeated stimuli was always upright, and the other was rotated $0^{\circ}, 30^{\circ}, 60^{\circ}, 90^{\circ}$, or $180^{\circ}$ from the upright. In Experiment 1 of the present study, we extend these conditions by presenting the stimuli in all combinations of $0^{\circ}, 90^{\circ}, 180^{\circ}$, and $270^{\circ}$, clockwise from the upright. This provides a more stringent test of the extent to which RB is independent of orientation. We did not include rotations of $30^{\circ}$ or $60^{\circ}$, since Harris and Dux's study suggested little difference between these rotations and that of $90^{\circ}$-and, if anything, rotations of $90^{\circ}$ produced slightly worse recognition. The use of letters rather than pictures tests the generality of the earlier findings. The upright is arguably especially important for letters, which are defined in terms of their upright orientations, and letters such as $\mathrm{b}$ and $\mathrm{d}$ change identity when rotated $180^{\circ}$. When letters are rotated away from the upright, they are named more slowly (Corballis, Zbrodoff, Shetzer, \& Butler, 1978; Jolicœur, Snow, \& Murray, 1987) and inaccurately (Jolicœur \& Landau, 1984). Although these effects are slight, they raise the possibility that both recognition and RB may be influenced by orientation under RSVP.

The use of letters also allows us to test, in Experiment 2, whether RB depends on the repetition of the name or the shape of a stimulus, by using the letters b, d, $\mathrm{p}$, and $\mathrm{q}$. These letters have the same basic shape but are differently named. Consequently, if RB is independent of orientation but depends on shape rather than name, then we might expect RB for any paired combination of these letters. On the other hand, Bavelier and Potter (1992) have shown that there is RB when the same letters are presented in different case, suggesting that the name rather than the shape of a letter determines RB. If this is so, then there should be no RB for combinations of $b$, $\mathrm{d}, \mathrm{p}$, and $\mathrm{q}$ that are named differently. There is reason to suppose, though, that the letters $b, d$, p, or q might be exceptional. For example, when they are rotated away from the upright, observers mentally rotate them to the upright in order to identify them (Corballis \& McLaren, 1984), whereas mental rotation does not seem to be involved in the identification of other letters (Corballis et al., 1978). Since Experiment 2 did indeed suggest that RB depended on the shape of the letters, and not on their names, we carried out a replication of Bavelier and Potter's (1992) result in Experiment 3.

\section{EXPERIMENT 1}

\section{Method}

Subjects. Fifteen subjects, 8 men and 7 women, volunteered. Their ages ranged from 19 to 35 years, with a mean age of 24.0 $(S D=4.36)$.

Stimuli. The stimuli consisted of RSVP streams of 7 items, presented at a rate of 10 items per sec in the center of the screen. The refresh rate of the screen was $60 \mathrm{cps}$, and each item was on the screen for four screen refreshes (approximately $67 \mathrm{msec}$ ), followed by two blank screens (approximately $33 \mathrm{msec}$ ). The items were either digits from 2 to 9 , or uppercase letters drawn from the set A, B, C, D, E, F, G, J, L, P, R, T, V, and Y, and each could appear in any one of the orientations $0^{\circ}, 90^{\circ}, 180^{\circ}$, or $270^{\circ}$, clockwise from upright. The experiment was programmed and controlled using Micro Experimental Laboratory Version 2 (MEL2) software (Schneider, 1995). The characters were presented in Mel font, a plain, sans serif font in which all of the lines are of equal width (in this case, $2 \mathrm{~mm}$ ). Each character was $13-\mathrm{mm}$ high and approximately $10-\mathrm{mm}$ wide when upright. The subjects viewed the screen from a distance of $57 \mathrm{~cm}$, so that each $\mathrm{cm}$ on the screen subtended $1^{\circ}$ of visual angle.

In each sequence, the subjects were asked to name the letters and ignore the digits. There were four kinds of sequences:

1. Nonrepeating sequences. Two digits were presented, followed by three letters, followed by two digits. All were different but otherwise randomly selected. The orientations of the digits and middle letter were randomly selected. The orientations of the first and third letters were systematically varied, with each of the 16 possible paired combinations presented 10 times, for a total of 160 trials. Rotated digits were presented simply as foils, rotated randomly to rule out any overall masking effects related to orientation.

2. Repeating sequences. The sequences were the same as in Item 1 except that the third letter was always the same as the first.

3. Two-letter Control A. The sequences were the same as in Item 1 except that the third letter was replaced by a digit presented in a random orientation. This digit was randomly selected, but different from the other digits. There were 10 trials for each orientation of the first letter, making 40 trials in all.

4. Two-letter Control B. The sequences were the same as in Item 3 except that the first letter was replaced by a digit presented in a random orientation.

One reason for the two control tasks was to provide conditions in which there were actually only two letters, so that subjects were not under the impression that there were always three letters. Hence, subjects were less likely to guess a third letter if they only saw two, especially in the repeating sequences.

Examples are shown in Figure 1. Altogether there were 400 trials, spread over two testing sessions, and presented in a randomized order.

Procedure. The subjects were instructed to watch the sequences and then type in the letters they saw, ignoring the digits. The programmed routine allowed them to type in no more than three letters. They were told that some letters might repeat, and if so, they should type in the repeated letter twice. They were also told that some sequences contained only two letters. If they saw fewer than three let-

$\begin{array}{llllllll}\text { Nonrepeating sequence: } & N & 6 & \searrow & \sqcup & \mathrm{T} & \infty & 4 \\ \text { Repeating sequence: } & 7 & G & \succ & \mathrm{P} & \prec & 3 & \sim \\ \text { Two-letter Control A: } & \nabla & 7 & < & \bullet & 6 & G & 3 \\ \text { Two-letter Control B: } & 8 & \mathcal{E} & \sim & \mathrm{G} & \Gamma & 8 & \curvearrowleft\end{array}$

Figure 1. Illustrative examples of sequences. The font shown here is similar but not identical to the one actually used in the experiments. 
Table 1

Mean Percentage of Trials on Which the Subjects Reported the First Letter in Control A and the Second Letter in Control B, As a Function of the Orientations of the Letters

\begin{tabular}{ccccc}
\hline & \multicolumn{4}{c}{ Orientation } \\
\cline { 2 - 5 } & $0^{\circ}$ & $90^{\circ}$ & $180^{\circ}$ & $270^{\circ}$ \\
\hline Control A & 85.8 & 88.9 & 83.7 & 86.3 \\
Control B & 82.6 & 88.9 & 86.3 & 88.4 \\
\hline
\end{tabular}

ters, they were to use the space bar to register blanks, but they were not discouraged from guessing if they were unsure.

The subjects were given 10 practice trials prior to the first session only, followed by the two sessions of 200 experimental trials. Each trial was triggered by pressing either the " 1 " or the "=" key. A fixation cross then appeared for $500 \mathrm{msec}$, followed by the sequence.

\section{Results}

Reported letters were scored correct if they were in the stimulus sequences, regardless of the order of report, except that a repeated letter had to be reported twice to be scored as correct on both occasions.

Report of individual letters in control sequences. In the two-letter control conditions, orientation had little effect on report of individual letters. For Control A, the effect of orientation on report of the first letter was not significant $[F(3,42)=0.66, p=.58]$, whereas for Control $\mathrm{B}$, the orientation of the second letter also had no significant effect on report $[F(3,42)=0.87, p=.46]$. The mean percentages of times the subjects correctly reported the first letter in Control A, and the second letter in Control B, are shown for each orientation in Table 1.

Repetition blindness: Report of first and third letters. The subjects reported both the first and the third letter correctly on $35.6 \%$ of nonrepeating sequences and on $19.8 \%$ of repeating sequences, a highly significant main effect $[F(1,14)=14.04, p=.002]$, strongly suggestive of $\mathrm{RB}$.

There were no significant effects of the angular orientations of the letters, nor any significant interactions between the orientations and whether or not the first and third letters were repeated. However, the interaction between the orientations of the first and third letters approached significance $[F(9,126)=1.80, p=.075]$, as did the triple interaction between the two orientations and the repetition factor $[F(9,126)=1.87, p=.063]$. The means for each combination of orientations of the first and third letters are shown for each condition in Table 2, along with the differences between conditions. The most striking feature of Table 2 is that accuracy was generally higher when orientations of the first and third letters were the same, but only when the first and third letters were repeated. This meant that the RB effect itself, reflected in the differences, was systematically less when the two orientations were the same than when they were different.

To test the effect of same versus different orientations, the absolute difference between the two orientations was treated as an independent variable constituting part of the interaction. The variance due to this difference was not significant $[F(2,126)=2.64$, n.s.], leaving an insignificant residual from the interaction $[F(7,126)=1.55$, n.s. $]$. However, the interaction between the difference and the repetition factor was significant $[F(2,126)=6.43, p<$ $.01]$, leaving an insignificant residual from the triple interaction $[F(7,126)=0.56$, n.s. $]$.

This interaction is plotted in Figure 2. For nonrepeating sequences, report of the first and third letters increased slightly with the angular distance between them, although the simple effect of angular distance was not significant $[F(2,28)=0.86, p=.43]$. For the repeating sequences, in contrast, report of the repeated letters decreased with angular distance $[F(2,28)=11.35, p<.001]$. That is, RB was actually less when the repeated letters were in the same orientation than when their orientations differed.

Report of the second (middle) letter. Report of the second letter (whose orientation varied randomly) was significantly higher for repeating sequences $(78.1 \%)$ than for nonrepeating sequences $(69.5 \%)[F(1,14)=16.78$, $p=.001]$, indicating a repetition advantage. There were no significant effects related to the orientations of the first and third letters.

\section{Discussion}

Angular orientation had little effect on the report of individual letters presented in rapid sequence. More importantly, there was little effect of orientation on RB, implying that early, and possibly preconscious, access to letter identity is orientation invariant. There was only one effect related to orientation: RB was less when the critical letters were in the same orientation than when they were in different orientations. Kanwisher et al. (1999) also found this to be so in their study of RB in recognition of objects, and suggested that repeated presentation of an identical item, even though separated by a different item, may induce "a certain kind of characteristic flicker" (p. 138) that

Table 2

Percentage of Trials on Which the First and Third Letters Were Both Correctly Reported in Experiment 1 As a Function of the Orientations of the Letters, Condition (Nonrepeated and Repeated Sequences), and Difference Between Conditions (Nonrepeated Minus Repeated)

\begin{tabular}{|c|c|c|c|c|c|c|c|c|c|c|c|c|}
\hline \multirow{3}{*}{$\begin{array}{c}\text { 1st Letter } \\
\text { Orientation }\end{array}$} & \multicolumn{12}{|c|}{ 3rd Letter Orientation } \\
\hline & \multicolumn{4}{|c|}{ Nonrepeated Condition } & \multicolumn{4}{|c|}{ Repeated Condition } & \multicolumn{4}{|c|}{ Difference } \\
\hline & $0^{\circ}$ & $90^{\circ}$ & $180^{\circ}$ & $270^{\circ}$ & $0^{\circ}$ & $90^{\circ}$ & $180^{\circ}$ & $270^{\circ}$ & $0^{\circ}$ & $90^{\circ}$ & $180^{\circ}$ & $270^{\circ}$ \\
\hline $0^{\circ}$ & 34.7 & 36.7 & 35.3 & 40.0 & 26.7 & 16.7 & 20.7 & 24.0 & 8.0 & 20.7 & 14.6 & 16.0 \\
\hline $90^{\circ}$ & 35.3 & 33.3 & 29.3 & 32.0 & 14.7 & 25.3 & 22.0 & 16.0 & 20.6 & 8.0 & 11.3 & 16.0 \\
\hline $180^{\circ}$ & 42.0 & 34.0 & 34.7 & 36.0 & 12.0 & 19.3 & 28.0 & 14.7 & 30.0 & 14.7 & 6.7 & 21.3 \\
\hline $270^{\circ}$ & 32.0 & 41.3 & 40.0 & 33.3 & 10.0 & 21.3 & 20.7 & 24.7 & 22.0 & 20.0 & 19.3 & 8.6 \\
\hline
\end{tabular}




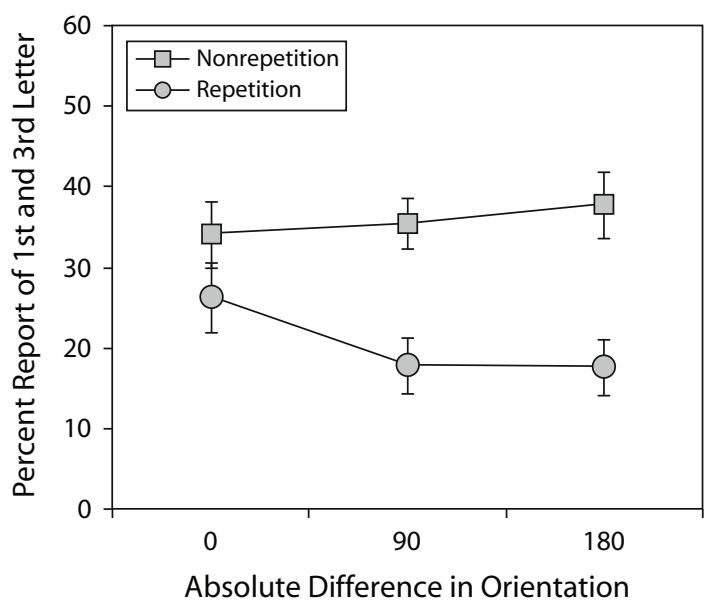

Figure 2. The percentage of trials on which subjects reported both the first and the third letter for nonrepeating and repeating sequences. Error bars are standard errors.

signals double presentation. An alternative possibility is that occasional registration of the orientation of the first letter may prime the attachment of the same orientation to the third letter. This is also suggested by evidence that preknowledge of orientation can improve the subsequent ease with which objects are recognized and named (Graf, Kaping, \& Bülthoff, 2005). In any event, the effect is the opposite of what one would predict if orientation is intrinsic to the recognition process, since repeating a letter in the same orientation is a more exact repetition than is repeating it in a different orientation.

Harris and Dux (2005), using pictures of objects as stimuli, reported a more complex interaction, in which RB was diminished when the first stimulus was rotated $180^{\circ}$ from upright and the third was upright. Our results show the reverse, if anything; the RB effect was in fact largest under these conditions. When the first stimulus was upright and the third rotated $180^{\circ}$, Harris and Dux found $\mathrm{RB}$ to depend on whether or not the second stimulus was rotated away from the upright; we were unable to test this in our experiment because the orientation of the second stimulus was randomly assigned and was not recorded.

The repetition advantage in report of the second (middle) letter suggests that repeated trials required less processing than nonrepeated trials, probably because only two rather than three types were activated, reducing the overall processing load. A similar effect was reported by Harris and Dux (2005).

\section{EXPERIMENT 2}

Is $\mathrm{RB}$ due to repetition of the same name, or to repetition of the same shape? Our finding that RB is invariant with respect to orientation allows us to distinguish these alternatives by presenting the letters $b, d, p$, and $q$. Thus, $b$ and $d$ have the same shape as $q$ and $p$, respectively, but are differently named. Further, $b$ and $p$ are simply mirror reflections of $\mathrm{d}$ and q, respectively, and there are reasons to suppose that the extraction of shape may be independent of reflection as well as of orientation (Corballis, 1988). Consequently, if shape is the critical attribute underlying $\mathrm{RB}$, we might expect RB for all possible pairs of these letters. As a control, we also presented the letters $\mathrm{t}$ and $\mathrm{k}$.

In this experiment, the letters were presented in only two orientations - upright and inverted.

\section{Method}

Subjects. Ten subjects, 5 men and 5 women, volunteered. Their ages ranged from 19 to 28 , with a mean age of $22.3(S D=2.79)$.

Stimuli. As in Experiment 1, the stimulus sequences consisted of 7 characters presented in rapid sequence, with each on the screen for four refresh screens (approximately $67 \mathrm{msec}$ ), followed by two blank screens (approximately $33 \mathrm{msec}$ ). Thus, the presentation rate was 10 characters per sec. The first 2 and last 2 characters were digits, randomly chosen, without replacement, from the series 2 through 9. Each was presented either upright or inverted, according to a random schedule. The middle 3 characters were lowercase letters. The second letter was chosen at random from the series a, c, e, $\mathrm{j}, \mathrm{r}, \mathrm{s}, \mathrm{v}, \mathrm{x}$, and $\mathrm{z}$.

The first and third letters were chosen either from the letters $b$ and $\mathrm{d}$, or from the letters $\mathrm{t}$ and $\mathrm{k}$. They were either repeated (i.e., $\mathrm{b}-\mathrm{b}$, $\mathrm{d}-\mathrm{d}, \mathrm{t}-\mathrm{t}$, and $\mathrm{k}-\mathrm{k}$ ) or nonrepeated (i.e., $\mathrm{b}-\mathrm{d}, \mathrm{d}-\mathrm{b}, \mathrm{t}-\mathrm{k}$, and $\mathrm{k}-\mathrm{t}$ ). Each letter of each pair was either upright or inverted, in each possible combination. It should be noted that $\mathrm{b}$ and $\mathrm{d}$, when inverted, are seen as $\mathrm{q}$ and $\mathrm{p}$, and subjects were expected to report them as $\mathrm{q}$ and $\mathrm{p}$, respectively. Nevertheless, they were constructed by inverting $b$ and $\mathrm{d}$, so as to avoid any other distinguishing features (such as a tail on the letter q). The eight pairs of letters combined with the four possible combinations of orientation made up 32 conditions, and each was presented five times to make a total of 160 trials.

The experiment was programmed using MEL2 software, and the characters were presented in the same font as was used in Experiment 1 . The letters b, d, p, q, t, and f were 13-mm tall, whereas the short letters, such as a and c, were 9-mm tall. The line thickness was $2 \mathrm{~mm}$.

Procedure. As in Experiment 1, the subjects were told simply to watch each sequence and then type in the letters they saw, ignoring the digits. They were warned that some of the letters were upside down. The computer routine allowed entry of up to three letters. The subjects were told that the letters were sometimes repeated, and to type in any repeated letter twice. If they saw fewer than three letters, they were to use the space bar to record blanks. The subjects were told that they might see any of the letters b, d, p, and q, in addition to $\mathrm{t}, \mathrm{k}$, and the intervening letters. Thus, there was no reason to expect them to interpret a $\mathrm{p}$ as an inverted $\mathrm{d}$, or a $\mathrm{q}$ as an inverted $\mathrm{b}$.

Subjects were given 10 practice trials before the 160 experimental trials.

\section{Results and Discussion}

Reported letters were scored correct if they were in the stimulus sequences, regardless of the order of report, except that a repeated letter had to be reported twice to be scored as correct on both occasions.

To assess RB, trials on which both the first and the third letter were correctly reported were tallied. The inverted $\mathrm{b}$ and $\mathrm{d}$ were recorded as correct if they were reported as $q$ and $p$, respectively. Repeated measures ANOVA was carried out, in which the factors were letter group (b-d vs. $t-k$ ), repetition, orientation (upright vs. inverted) of the first letter, and orientation of the third letter. For the $\mathrm{b}-\mathrm{d}$ group, $\mathrm{b}$ and $\mathrm{q}$ were regarded as repeated, as were $\mathrm{d}$ and $\mathrm{p}$. There was again a significant effect of repetition, with nonrepeated pairs reported much more accurately than repeated ones $[F(1,9)=30.94, p<.001]$, and $\mathrm{t}-\mathrm{k}$ 
Table 3

Percentage Report of Both First and Third Letters in Experiment 2 As a Function of Pairs, Orientation of the Third Letter, and Repetition

\begin{tabular}{cccccc}
\hline & \multicolumn{2}{c}{$\mathrm{b}-\mathrm{d}$ Pairs } & & \multicolumn{2}{c}{$\mathrm{t}-\mathrm{k}$ Pairs } \\
\cline { 2 - 3 } \cline { 5 - 6 } Orientation & Repeated & Nonrepeated & & Repeated & Nonrepeated \\
\hline $0^{\circ}$ & 13.5 & 10.5 & & 13.5 & 45.0 \\
$180^{\circ}$ & 11.5 & 14.0 & & 11.5 & 43.0 \\
\hline
\end{tabular}

pairs were reported significantly more accurately than $b-d$ pairs $[F(1,9)=18.70, p=.002]$. More importantly, the interaction between the two was also highly significant $[F(1,9)=15.12, p=.004]$.

The only significant effect of orientation was a triple interaction between letter group, repetition, and orientation of the third letter $[F(1,9)=6.44, p<.032]$. This is shown in Table 3. For b-d pairs, the inverted letters were seen as the upright letters $q$ and $\mathrm{p}$, respectively, whereas for $\mathrm{t}-\mathrm{k}$ pairs, they were seen as inverted ts and ks, respectively. The interaction therefore has little to do with orientation per se, and since inversion has different consequences for the two pairings, separate ANOVAs were conducted for each pairing. Table 3 shows that report of $t-k$ pairs was slightly better overall when the third letter was upright than when it was inverted, but this effect did not approach significance $[F(1,9)=0.63, p=.448]$. There was, however, a highly significant effect of repetition $[F(1,9)=22.96, p=.001]$. For the $\mathrm{b}-\mathrm{d}$ pairs, there appears to be an interaction between orientation of the third letter and repetition, with more accurate report when the third letter was upright (b or d) when repeated, but more accurate when it was inverted (q or $\mathrm{p}$ ) when not repeated. This may simply reflect more accurate report of some pairings than of others, but the interaction was not, in fact, significant $[F(1,9)=2.66, p=.137]$, nor were any of the other effects or interactions.

Low performance on the $b-d$ pairs raises the question of whether the subjects simply had difficulty seeing these letters at all. However, the subjects responded on $b-d$ trials with at least one of the letters $b, d, p$, and q, whether correctly or not, on $94.3 \%$ of trials, which is almost identical to the $94.0 \%$ of $\mathrm{t}-\mathrm{k}$ trials in which they responded with at least one of the letters $t$ and $k$. Their overall accuracy of reporting these letters on b-d pairs, however, was $43.5 \%$, in comparison with $59.7 \%$ accurate report of $\mathrm{t}$ and $\mathrm{k}$ in $\mathrm{t}-\mathrm{k}$ trials. This difference might reflect, in part, the fact that chance responding would have favored $t-k$ pairs, since there were only two alternatives, in comparison with four, among $b, d, p$, and q. Nevertheless, the high frequency of reporting at least one from among the $\mathrm{b}-\mathrm{d}-\mathrm{p}-\mathrm{q}$ set, suggests that subjects were able to discern the basic shape of these letters, but had difficulty distinguishing one from another.

Figure 3 plots the percentage of trials on which both the first and third letters were correctly reported, as a function of pairs, repetition, and the difference in angular orientation. Although ANOVA, with pairs, repetition, and angular difference as factors, revealed no significant effects of angular difference, the means plotted in Figure 3 indicate a number of trends. For $\mathrm{t}-\mathrm{k}$ pairs, $\mathrm{RB}$ was in fact slightly less when the letters were repeated in the same orientation than in different orientations, as in Experiment 1, although the difference here was not significant $[F(1,9)=$ $1.55, p=.245]$. That is, RB may be slightly less when the same shape is repeated in the same orientation than when it is repeated in different orientations.

For $\mathrm{p}-\mathrm{q}$ pairs, the pattern of results can be described in terms of the geometric relations among the presented letters (see also the caption to Figure 3). Report of the first and third letters was in fact worst (9\%) when the letters were repeated in the same orientation - that is, when the letters were in fact identical (b-b, d-d, p-p, q-q). For nonrepeated letter shapes in the same orientation, accuracy rose to $11.5 \%$, and here the presented pairs were in fact left-right mirror images ( $b-d, d-b, p-q, q-p)$. For repeated letter shapes in different orientations, it rose further to $16.0 \%$, and here the letter pairs were rotations $(b-q$, $d-p, q-b, p-d)$. Finally, for different letter shapes in different orientations, accuracy was $13.0 \%$, and the letters were in this case inversions (b-p, d-q, p-b, q-d). Although the analysis of accuracy for $b-d$ pairs alone revealed no significant effects of repetition or same versus different orientation, these means suggest that RB may not have been wholly independent of relative shapes and names. In this case, then, contrary to the results of Experiment 1, repetition of the same shape in the same orientation seems to lead to greater RB, if anything, than repetition in different orientations. The likely reason for this is that changing the orientations of the shapes here changed their names, which may have alleviated the RB.

Types of errors for $b-\mathbf{d}$ pairs. In order to gain some impression of confusions among the $b-d$ pairs, we classified confusion errors. We ignored all reported letters not

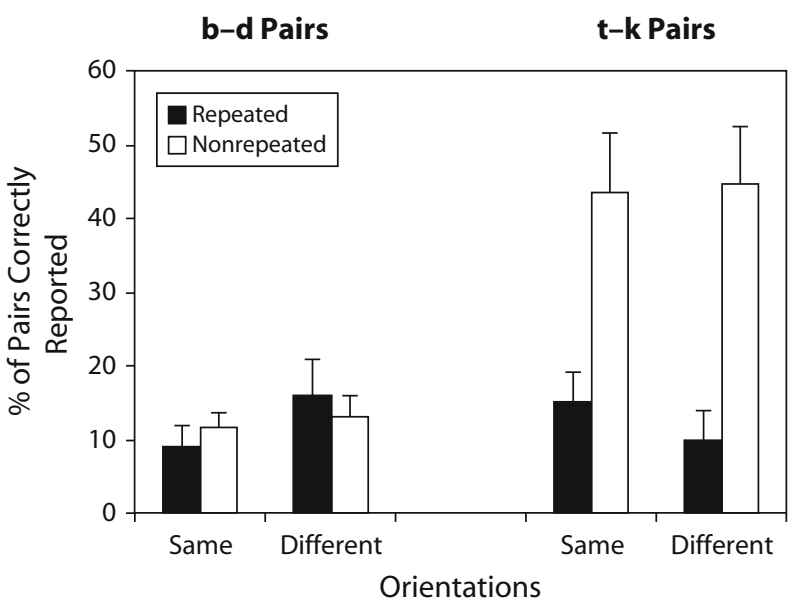

Figure 3. The percentage of trials on which subjects correctly reported $b-d$ and $t-k$ pairs in Experiment 2, as a function of whether their orientations were the same or different. Error bars are standard errors. For b-d pairs, reading from left to right on the graph, the relations between the two presented letters can be described as follows: same repeated letters are identical $(b-b, d-d$, $\mathrm{p}-\mathrm{p}, \mathrm{q}-\mathrm{q})$, same nonrepeated letters are left-right mirror images $(b-d, d-b, p-q, q-p)$, different repeated letters are rotations $(b-q$, $\mathrm{d}-\mathrm{p}, \mathrm{q}-\mathrm{b}, \mathrm{p}-\mathrm{d})$, and different nonrepeated letters are inversions (b-p, d-q, p-b, q-d). 
from the set $\mathrm{b}, \mathrm{d}, \mathrm{p}$, and $\mathrm{q}$, and restricted analysis to those cases in which the errors could be classified unambiguously: (1) if two different letters were presented (e.g., b-d), and one of two responses matched (e.g., b-q), we considered the incorrect response to be confused with the letter that was not reported correctly (e.g., d-q); (2) if the same letter was repeated (e.g., b-b), and the response included just one incorrect letter (e.g., d), or if it repeated a single incorrect letter (e.g., d-d), we considered the incorrect letter to be confused with the repeated stimulus letter. We ignored cases in which different letters were presented (e.g., b-d) and a single response matched neither of them (e.g., q), or if a double response matched neither of them (e.g., p-q), since the confusion could not be unambiguously classified.

Pairs of letters confused were classified as left-right reversals $(\mathrm{b}-\mathrm{d}, \mathrm{p}-\mathrm{q})$, rotations $(\mathrm{b}-\mathrm{q}, \mathrm{d}-\mathrm{p})$, or inversions (b-p, d-q). The numbers in each category were counted for each repetition condition for each subject, and subjected to ANOVA. There were significantly more errors under the repetition condition $[F(1,9)=9.29, p=.014]$, probably because errors under this condition could be less ambiguously classified. There was a significant difference between the three types of confusion $[F(2,18)=3.96, p=.038]$, but the interaction between repetition and confusion type was not significant $[F(2,18)=0.62, p=.549]$, suggesting that the pattern of confusions was not some artifact of repetition. Orthogonal contrasts showed that the mean number of left-right confusions (3.70) was significantly greater than the number of inversion (2.25) or rotation (2.00) confusions $[F(1,9)=12.15, p=.007]$, which did not differ significantly from each other $[F(1,9)=0.108, p=.750]$.

The predominance of left-right confusions is consistent with evidence from both animals and humans (especially children) that left and right are more easily confused than up and down (see Corballis \& Beale, 1976, for a review). It is also possible that the effect is purely perceptual, and that subjects could more easily detect whether the vertical line extended above or below the rounded portion of the letter than whether the rounded portion was to the left or right. Such an effect might be unrelated to left and right per se.

Summary. In summary, then, the poor report of b-d pairs suggests that RB depended mostly on the shapes of the letters, and not on their names - although there was some suggestion that RB was alleviated slightly when the pairs had different names. Subjects had great difficulty accurately reporting more than one of any of the paired combinations of $\mathrm{b}, \mathrm{d}, \mathrm{p}$, and $\mathrm{q}$, and an equal difficulty reporting repetitions of $t$ or of $k$, regardless of their orientations. There was no such difficulty when $t$ was paired with $\mathrm{k}$. The problems with $\mathrm{b}, \mathrm{d}, \mathrm{p}$, and q were slightly more evident when the pairs were left-right mirror images of each other (b-d or $p-q)$, suggesting that shape is extracted independently of both angular orientation and parity.

\section{EXPERIMENT 3}

Experiment 2 suggested that RB depends on the shapes of letters rather than their names. This result contradicts that of Bavelier and Potter (1992), who found that RB occurred when letters were repeated in different case. Ex- periment 3 was therefore designed to replicate this result, using the same basic parameters as were used in Experiments 1 and 2 .

\section{Method}

Subjects. Eleven subjects, 8 women and 3 men, volunteered. They were between 19 and 29 years old, with a mean age of 21.1 years.

Stimuli. As in the previous two experiments, the stimulus sequences consisted of 7 characters presented in rapid sequence, with each on the screen for four refresh screens (approximately $67 \mathrm{msec}$ ), followed by two blank screens (approximately $33 \mathrm{msec}$ ). Thus, the presentation rate was 10 characters per sec. The first 2 and last 2 characters were digits, randomly chosen, without replacement, from the series 2 through 9 . The middle three stimuli were letters drawn from the pool A, B, D, E, F, G, H, Q, R, and T, and their lowercase equivalents. The three letters could appear either in uppercase or lowercase. All characters were again presented in the same font as was used in the previous two experiments, and no attempt was made to equate uppercase and lowercase letters for size. Thus, uppercase letters were $13-\mathrm{mm}$ high, as were tall lowercase letters, whereas short lowercase letters were 9-mm high. Within each combination, the third letter had either the same name as the first (repetition) or a different name (nonrepetition); the second letter was always different. The combinations of letter case and repetition made up a total of 16 combinations, each presented six times, making a total of 96 trials. On each trial, the letters were chosen randomly, with the exception that on a repetition trial, the first and third letters were the same, and the trials were randomized across conditions. All of the letters were upright.

The experiment was programmed and controlled using MEL2 software.

Procedure. As in the previous experiments, the subjects were told simply to watch each sequence and then type in the letters they saw, ignoring the digits. The computer routine allowed entry of up to three letters. The subjects were told that the letters were sometimes repeated, and to type in any repeated letter twice. If they saw fewer than three letters, they were to use the space bar to record blanks. The subjects were given 12 practice trials, with conditions randomly chosen, before the 96 experimental trials.

\section{Results and Discussion}

Reported letters were again scored correct if they were in the stimulus sequences, regardless of the order of report, except that a repeated letter had to be reported twice to be scored as correct on both occasions.

ANOVA was carried out on the number of trials in which both first and third letters were reported correctly, as a function of repetition, the case of the first letter, the case of the second letter, and the case of the third letter. This revealed a highly significant effect of repetition $[F(1,10)=37.85$, $p<.001$ ], but no significant effects related to the case of the first or third letters, nor their interaction, nor their interactions with repetition. In particular, the triple interaction between repetition and the cases of the first and third letters did not approach significance $[F(1,10)=0.342, p=.572]$. This suggests that the repetition effect did not depend on the cases of the first and third letters, or on whether they were the same or different. The means for the different combinations of case are shown in Table 4, and it is clear that the repetition effect was robust for all combinations of case.

The case of the second letter showed a significant interaction with the case of the third letter $[F(1,10)=7.86$, $p=.019]$. When the second letter was uppercase, report of both first and third letters was better when the third letter was also in uppercase $(36.7 \%$ correct) than when it 
Table 4

Percent Correct Report of Both First and Third Letters in Experiment 3, As a Function of the Case of the Letters and of Repetition

Case Combinations

(1st Letter/3rd Letter)

\begin{tabular}{lllll} 
& \multicolumn{4}{c}{ (1st Letter/3rd Letter) } \\
\cline { 2 - 5 } Condition & $\mathrm{U} / \mathrm{U}$ & $\mathrm{U} / \mathrm{L}$ & $\mathrm{L} / \mathrm{U}$ & $\mathrm{L} / \mathrm{L}$ \\
\hline Nonrepeated & 59.1 & 45.5 & 50.8 & 47.7 \\
Repeated & 19.7 & 20.5 & 12.1 & 17.4
\end{tabular}

Note-U, uppercase; L, lowercase.

was in lowercase $(29.9 \%)$, but when it was in lowercase, this was (marginally) reversed ( $34.1 \%$ vs. $35.6 \%)$. It is not clear what might explain this result, but the case of the second letter otherwise gave rise to no significant effects.

The strong repetition effect, regardless of the case of the letters, confirms Bavelier and Potter's (1992) finding that RB occurs whether or not the letter is repeated in the same or different case. Contrary to the implications of Experiment 2, then, RB can occur at the level of name even when the shapes of the repeated letters are different.

\section{GENERAL DISCUSSION}

The results of Experiments 1 and 2, and those of Harris and Dux (2005) and Kanwisher et al. (1999), suggest that early registration of an object's identity is dissociable from perception of its orientation. This is consistent with the view that orientation is a circumstantial attribute that may help to tokenize one episodic appearance of an object as distinct from another, and is not part of an object's type (cf. Corballis, 1988; Kanwisher, 1987). This may explain why some patients with neurological damage are able to recognize objects correctly, but are unable to determine their orientations (Fujinaga et al., 2005; Harris et al., 2001; Karnath et al., 2000; Turnbull et al., 2002). Logically, in fact, the perception of orientation must come after the perception of shape or name, since observers cannot know the orientation of a shape as a shape until they know what the shape actually is. Thus, the identity of a shape is presumably extracted by the ventral (or "what") stream, whereas orientation depends on information extracted by the dorsal (or "where") stream of the cortical visual system (Ungerleider \& Mishkin, 1982). To perceive a shape in its presented orientation must presumably depend on some kind of interaction between these two streams. This mapping is presumably attention demanding (cf. Treisman, 1988), time consuming, and greatly attenuated under RSVP.

Experiment 1 also confirms Kanwisher et al.'s (1999) finding that RB is less pronounced when the stimuli are repeated in the same orientation than when they are oriented differently. This might be attributed to priming, so that a given stimulus is better perceived if repeated in the same orientation than if repeated in a different orientation. This effect is still not sufficient, however, to counteract the effect of repetition itself. Further, it might be taken to imply that orientation is processed, at least to some level, because otherwise, RB should be independent of the relative orientations of the stimuli. However, if the effect were dependent on perception of orientation, one might have expected it to be stronger when the letters were upright than when they were inverted, since perceived orientation should be more salient for upright than for rotated letters. Table 2 shows, however, that the priming effect is approximately constant over the different orientations. This suggests that the effect does not depend on perceived orientation of the letter itself, but may depend, rather, on repetition of the same proximal stimulus.

The letters $b, d, p$, and q pose a special problem, because their orientations must be specified before their separate identities can be established. Experiment 2 showed that RB occurred when any one of these letters was followed by any other in RSVP - that is, they were treated as having the same shape. This is consistent with the approach taken by Corballis (1988), who proposed that the initial registration of shape is independent of any reference frame, and depends instead on descriptions internal to the shape itself. Such descriptions make no reference to directions such as up, down, left, or right. For example, the letters $b, d, p$, and $q$ all meet the description of a curve attached to a straight line, with one end of the curve meeting one end of the line and the other joining approximately halfway along it. Such descriptions apply equally to mirror-image shapes (enantiomers), which might explain why there is RB between the mirror-image pairs b-d and p-q.

In real-life settings, objects appear in arbitrary orientations. The letters $b, d, p$, and $q$ somewhat resemble a primitive hand ax, with a sharpened slab of stone hafted to one end of a wooden handle. The viewer might see this object in any orientation, or in either of two mirror-image forms, depending on which side faces him or her. In short, the same actual object finds equal resemblance in any one of the four letters $b, d, p$, and q. This is not to say that our perception of these letters has anything to do with hand axes; the point is simply that we have evolved mechanisms of shape constancy that enable us to see the same objects in different orientations and locations, and these mechanisms can influence the way in which we perceive simple shapes. These mechanisms create difficulty, however, in identifying shapes, such as the letters b, d, p, and q, that depend on orientations for their very definition. This might explain why animals and people, especially children, often have difficulty distinguishing among them, and among mirrorimage shapes in general (see Corballis \& Beale, 1976, for a review). Indeed, confusions among the letters $b, d, p$, and $\mathrm{q}$ are among the most common errors that children make when learning to read, and are often a source of difficulty in reading disability (see, e.g., Terepocki, Kruk, \& Willows, 2002).

Although Experiment 2 suggested that RB can occur when the stimuli differ in name but share the same basic shape, Experiment 3 replicated Bavelier and Potter's (1992) converse finding that RB can occur when the repeated letter has the same name but a different shape. One conclusion that might be drawn from these somewhat conflicting results is that RB can occur either at the level of shape or at the level of name. Indeed, there was some sug- 
gestion from Experiment 2 that RB among the letters $b$, $\mathrm{d}, \mathrm{p}$, and $\mathrm{q}$ was alleviated when the repeated letter shapes had different names, although the effect was not statistically significant. Because the letters b, d, p, and q require orientation (and left-right sense) to be specified before they can be named, subjects evidently have difficulty either unambiguously naming them or tokenizing them as separate "events" in RSVP, and RB occurs at the level of shape information.

Some authors have suggested that $\mathrm{RB}$ results, not from a dissociation between type and token, as suggested by Kanwisher (1987), but rather from a failure of retrieval (e.g., Armstrong \& Mewhort, 1995; Fagot \& Pashler, 1995 ) or of online reconstruction (Whittlesea \& Masson, 2005) or integration (Morris \& Harris, 2002). Our results do not clearly distinguish between these different views, although the fact that identity and orientation can be dissociated neuropsychologically as well as in RB suggests that the effect is probably one of registration rather than retrieval. In the case of letters, moreover, RB does not seem to depend on forgetting of the repeated stimulus, nor on guessing, output interference, response bias, or temporal overlap in processing (Park \& Kanwisher, 1994), suggesting that the problem is fundamentally one of binding orientation to identity, whether or not this is part of a more general process of tokenization.

In summary, our results suggest that RB occurs prior to the perception of orientation. In the case of familiar shapes such as letters, it may occur at the level of naming. In the case of the exceptional letters $b, d, p$, and $q$, naming requires the perception of orientation, so that subjects appeared generally unable even to name these letters under RSVP, and RB occurred at the level of shape. More generally, the results offer continued support for the view that shape is extracted independently of orientation, and is generally sufficient to permit the shape to be identified and named. Nevertheless, there are some important exceptions. Orientation-free descriptions fail to distinguish mirror images, such as the distinctions between $\mathrm{b}$ and $\mathrm{d}, \mathrm{p}$ and $\mathrm{q}$, or a left and a right hand, and they probably also fail to capture subtle relational cues. For example, familiar faces are very difficult to recognize when inverted (Valentine, 1988), and inverted handwriting is almost impossible to read (Rock, 1973). Orientation-free descriptions may be relatively crude, but sufficient to provide at least a basic-level description, and correction for orientation may be required for more detailed processing (cf. Corballis, 1988).

\section{AUTHOR NOTE}

This research was supported by a grant from the Auckland University Research Committee. We thank Irina Harris and Paul Dux for general discussion, Steve Palmer for discussion leading to Experiment 2, and Min Cheng for help in running Experiment 3. Correspondence concerning this article should be addressed to M. C. Corballis, Department of Psychology, University of Auckland, Private Bag 92019, Auckland 1001, New Zealand (e-mail: m.corballis@auckland.ac.nz).

\section{REFERENCES}

Armstrong, I. T., \& Mewhort, D. J. K. (1995). Repetition deficit in RSVP displays: Encoding failure or retrieval failure? Journal of Experimental Psychology: Human Perception \& Performance, 21, 1044-1052.
Bavelier, D., \& Potter, M. C. (1992). Visual and phonological codes in repetition blindness. Journal of Experimental Psychology: Human Perception \& Performance, 18, 134-147.

Biederman, I. (1987). Recognition-by-components: A theory of human image understanding. Psychological Review, 94, 115-147.

Biederman, I., \& Gerhardstein, P. C. (1993). Recognizing depthrotated objects: Evidence and conditions for three-dimensional viewpoint invariance. Journal of Experimental Psychology: Human Perception \& Performance, 19, 1162-1182.

Bülthoff, H. H., \& Edelman, S. (1992). Psychophysical support for a two-dimensional view interpolation theory of object recognition. Proceedings of the National Academy of Sciences, 89, 60-64.

Corballis, M. C. (1988). Recognition of disoriented shapes. Psychological Review, 95, 115-123.

Corballis, M. C., \& Beale, I. L. (1976). The psychology of left and right. Hillsdale, NJ: Erlbaum.

Corballis, M. C., \& McLaren, R. (1984). Winding one's $p$ s and $q$ s: Mental rotation and mirror-image discrimination. Journal of Experimental Psychology: Human Perception \& Performance, 10, 318-327.

Corballis, M. C., Zbrodoff, N. J., Shetzer, L. I., \& Butler, P. B. (1978). Decisions about identity and orientation of rotated letters and digits. Memory \& Cognition, 6, 98-107.

Deutsch, J. A. (1962). A system for shape recognition. Psychological Review, 69, 492-500.

Fagot, C., \& Pashler, H. (1995). Repetition blindness: Perception or retrieval failure? Journal of Experimental Psychology: Human Perception \& Performance, 21, 275-292.

Fujinaga, N., Muramatsu, T., Ogano, M., \& Kato, M. (2005). A 3 -year follow-up study of "orientation agnosia." Neuropsychologia, 43, 1222-1226.

Graf, M., KaPING, D., \& BÜLThOFF, H. H. (2005). Orientation congruency effects for familiar objects: Coordinate transformations in object recognition. Psychological Science, 16, 214-221.

HARRIS, I. M., \& DUX, P. E. (2005). Orientation-invariant object recognition: Evidence from repetition blindness. Cognition, 95, 73-93.

Harris, I. M., Harris, J. A., \& CAINE, D. (2001). Object orientation agnosia: A failure to find the axis? Journal of Cognitive Neuroscience, 13, 800-812.

JolicceUR, P. (1985). The time to name disoriented natural objects. Memory \& Cognition, 13, 289-303.

Joliceur, P. (1990). Identification of disoriented objects: A dualsystems theory. Mind \& Language, 5, 387-410.

Jolicceur, P., \& LANDAU, M. J. (1984). Effects of orientation on the identification of simple visual patterns. Canadian Journal of Psychology, 38, 80-93.

Jolicceur, P., Snow, D., \& Murray, J. (1987). The time to identify disoriented letters: Effects of practice and font. Canadian Journal of Psychology, 41, 303-316.

Kanwisher, N. G. (1987). Repetition blindness: Type recognition without token individuation. Cognition, 27, 117-143.

Kanwisher, N. [G.], Yin, C., \& WoJCIUlik, E. (1999). Repetition blindness for pictures: Evidence for the rapid computation of abstract visual descriptions. In V. Coltheart (Ed.), Fleeting memories: Cognition of brief visual stimuli (pp. 119-150). Cambridge, MA: MIT Press.

Karnath, H.-O., Ferber, S., \& BülthofF, H. H. (2000). Neuronal representation of object orientation. Neuropsychologia, 38, 1235-1241.

Milner, A. D., \& Goodale, M. A. (1995). The visual brain in action. New York: Oxford University Press.

Morris, A. L., \& HARRIS, C. L. (2002). Sentence context, word recognition, and repetition blindness. Journal of Experimental Psychology: Learning, Memory, \& Cognition, 28, 962-982.

MurRay, J. E. (1995). The role of attention in the shift from orientationdependent to orientation-invariant identification of disoriented objects. Memory \& Cognition, 23, 49-58.

Palmer, S. E., Rosch, E., \& Chase, P. (1981). Canonical perspective and the perception of objects. In J. [B.] Long \& A. [D.] Baddeley (Eds.), Attention and performance IX (pp. 135-151). Hillsdale, NJ: Erlbaum

Park, J., \& Kanwisher, N. [G.] (1994). Determinants of repetition blindness. Journal of Experimental Psychology: Human Perception \& Performance, 20, 500-519.

Rock, I. (1973). Orientation and form. New York: Academic Press. Schneider, W. (1995). Micro Experimental Laboratory, Professional 
Version 2.0 [Computer software]. Pittsburgh, PA: Psychology Software Tools.

TARR, M. J., \& Pinker, S. (1989). Mental rotation and orientationdependence in shape recognition. Cognitive Psychology, 21, 233-282.

Terepocki, M., KruK, R. S., \& Willows, D. M. (2002). The incidence and nature of letter orientation errors in reading disability. Journal of Learning Disabilities, 35, 214-233.

Treisman, A. (1988). Features and objects: The Fourteenth Bartlett Memorial Lecture. Quarterly Journal of Experimental Psychology, 40A, 201-237.

Turnbull, O. H., Della Sala, S., \& Beschin, N. (2002). Agnosia for object orientation: Naming and mental rotation evidence. Neurocase, 8, 296-305.
Ungerleider, L. G., \& MishKIN, M. (1982). Two cortical visual systems. In D. J. Ingle, M. A. Goodale, \& R. J. W. Mansfield (Eds.), Analysis of visual behavior (pp. 549-586). Cambridge, MA: MIT Press.

VAlentine, T. (1988). Upside-down faces: A review of the effect of inversion upon face recognition. British Journal of Psychology, 79, 471-491.

Whittlesea, B. W. A., \& Masson, M. E. J. (2005). Repetition blindness in rapid lists: Activation and inhibition versus construction and attribution. Journal of Experimental Psychology: Learning, Memory, \& Cognition, 31, 54-67.

(Manuscript received July 26, 2005;

revision accepted for publication December 16, 2005.) 